\title{
Electoral competition and political rents
}

\author{
Michele Polo \\ IGIER and Bocconi University \\ Milan - Italy*
}

December 22, 1998

\begin{abstract}
We analyze the relation between the intensity of electoral competition and the dissipation of political rents. In a model with perfectly informed and heterogeneous voters, two candidates commmit to electoral platforms under a majority voting and winner-takes-all rule. If the proposed tax revenues exceed the cost of the public good, the winning candidate retains the surplus (political rents). The candidates are uncertain about voters' preferences. If they do not know the mean of voters' distribution (aggregate uncertainty), competition is relaxed and rents are positive. We then consider some extensions, as ideological positioning, increasing the number of candidates and imperfect commitment to the annouced policies.
\end{abstract}

Keywords: Electoral competition, political rents, probabilistic voting JEL classification numbers: $\mathrm{H} 1, \mathrm{H} 2, \mathrm{H} 4$.

\section{Introduction}

In this paper we tackle a problem which is common in the Public Choice literature, that is how the supply of a public good and the tax revenues to cover its cost are determined as the outcome of electoral competition.

\footnotetext{
*I'm greatly indebted with Michele Grillo in developing my ideas on political competition. I also benefited from long discussions with Michael Castanheira, Guido Tabellini. Thanks to Giuseppe Bertola, Eliana La Ferrara and Fausto Panunzi for useful comments on an earlier version. Financial support from Bocconi University is gratefully acknowledged.
} 
This apparently simple problem involves a complex interplay among different conflicts of interests and agency relations. First of all, there is a conflict of interests among different groups of voters on the content of redistributive policies. Second, there is an obvious conflict of interests among the candidates, the very engine of electoral competition, as they gain some utility from winning the office. Finally, a conflict exists between the electorate and the candidates which derives from the possibility that the latter, once elected, retain political rents.

There exists a wide literature on the features of electoral competition and the redistributive policies that are implemented in equilibrium ${ }^{1}$ : it mainly considers the first two conflicts of interest, that is the features of equilibrium platforms when rival candidates compete for the votes of a heterogeneous electorate, and its most celebrated result is the Median Voter Theorem. Although the Chicago School has claimed that electoral competition and democracy tend to produce efficient results ${ }^{2}$, few analytical research has explored the third problem, in which the elected can exploit their position to extract rents from office.

This latter topic can be addressed in two complementary ways, distinguishing between ex-ante and ex-post incentives: the former operate when electoral competition takes place, inducing the candidates to offer rentminimizing platforms to please the voters. Post-election incentives, instead, are related to the separation of powers and the checks and balances which characterize the political process after the elections, and influences the implementation of the policies and the rents realized ${ }^{3}$. These complementary levels of analysis have a point in common: they both address the issue of which are the rules and the conditions under which delegating the design and implementation of the policies to a group of agents (the politicians) can minimize the agency costs for the principals (the electorate).

In this paper we explore the ex-ante component of the story, studying under which conditions electoral competition minimizes political rents. There are two basic references for our work. The first is Stigler's (1972) contribution on the relation between economic and political competition. Stigler argued that, though economists agree that competition is a social mechanism aimed at reducing the unnecessary return to producers, surprisingly this role for competition has been almost neglected in the spatial theory of

\footnotetext{
${ }^{1}$ See, among others, Persson and Tabellini (1998a), part I and III, and Persson and Tabellini (1998b).

${ }^{2}$ See Wittman (1989).

${ }^{3}$ See Persson, Roland and Tabellini (1997).
} 
voting ${ }^{4}$. A possible step in this direction can be found in Donald Wittman's works $(1973,1977,1990)$, in which an income distribution game is studied where candidates are utility maximizers and participate in the division of the "pie". More recently, Grillo and Polo (1993), Myerson (1993b), Svennson (1997) and Persson and Tabellini (1998b) analyze the emergence of political rents in electoral games.

We set up a very simple model with heterogeneous voters, who have to elect a politician to provide a public good. Voters and candidates are utility maximizers. Two candidates compete for the office by committing to electoral platforms which specify the amount of the public good and the level of taxation. We do not impose any balanced budget constraint to the platforms, contrary to what is usually done in the literature ${ }^{5}$. In our setting, if the tax revenues are greater than the cost of the public good, the surplus is kept by the candidate in office. Since the candidates are utility maximizers, they design the platform in order to maximize their expected rents.

Voters' preferences can be separated in two components: the first, which is common to all voters and is known by the candidates, depends on the policy parameters, i.e. the tax rate and the supply of the public good. The second describes voters' heterogeneity, is not observed by the candidates and differs across voters with a common bias that shifts the mean of their distribution.

In this framework we investigate whether political competition induces efficient outcomes, i.e. the minimization of political rents. We exclude by assumption that the candidates (agents) are better informed than the voters (principals) about, for instance, the cost of the public good, i.e. in our model there is no room for informational rents of the politicians. In other words, if the candidates are able to retain some surplus, this is not due to their ability to exploit superior information, but to imperfect electoral competition. In this environment, candidates' competition for office might induce a dissipation of political rents (the choice of balanced budget platforms),

\footnotetext{
${ }^{4}$ It is now common in the literature - see Arrow (1990) and Myerson (1993a, 1993b) to observe that the spatial theory of voting builds on the first part of Hotelling's seminal contribution which studied competition between two firms that first choose location and then select a price. While Arrow (1990, p.ix) plainly states that in models of political competition "there is no equivalent of the price competition", Myerson (1993a, p.239) suggests that "levels of corruption and patronage may be competitive variables that are analogous to the price in Hotelling's economic model".

${ }^{5}$ See for instance Laffont (1989), p.51-53 and Lindbeck and Weibull (1987).
} 
which are more appealing for the voters. On the other hand, political rents can emerge if electoral competition is not sufficiently intense.

We find that the intensity of competition is deeply influenced by the nature of candidates' uncertainty on voters' preferences. If the candidates know the mean (and median) of the voters' distribution, they know which is the median platform and compete à la Bertrand, with complete rent dissipation. If, however, candidates have aggregate uncertainty on the mean (and median) of the voters' distribution, i.e. they don't know which is the median platform, competition is relaxed and positive rents emerge in equilibrium.

We consider then some extensions of the basic model. First we add a third variable to the electoral platforms, a "location" of the candidate on the ideological spectrum: now the candidates can influence also the idiosyncratic component of voters' preferences. We show that convergence to the median voter's ideological position still holds, while rents remain positive in case of aggregate uncertainty. In this example, the conflict of interest among voters (the median voter result) interacts with that between the electorate and the politicians (the positive rents result).

Our second extension pertains the increase in the number of candidates, which leads to a reduction of the equilibrium rents, suggesting a possible way out of our dilemma. Finally, we remove the assumption of candidates' perfect commitment to the promised platform. If an elected politician can cheat on the promised platform, although renouncing to be elected again, he trades off higher rents today with future rents if re-elected. Hence, a platform excessively moderate in rents is not believed by voters, which expect to be exploited by the elected politician. This additional constraint adds a second source of rents in the policy game: depending on the parameters of the model, actual rents will be determined by weak electoral competition or candidates moral hazard. Interestingly, as the number of candidates increases the rents required to prevent candidates' moral hazard increase, since a honest politician will be re-elected with a lower probability. Hence, increasing the number of candidates reduces the rents associated to imperfect electoral competition but makes the moral hazard constraint binding, preventing complete political rents dissipation.

The paper is organized as follows: in section 2 the main features of the basic model are presented; two versions are then discussed and the corresponding equilibria analyzed in section 3 and 4, distinguishing different degrees of uncertainty of the candidates. In section 5 three extensions are 
discussed: ideological positioning, $n$ canadidates and imperfect commitment. Concluding remarks follow.

\section{The model}

We assume there exist $I$ voters $(i=1, \ldots, I)$ with $I$ very large, and two candidates; electoral competition with more than two candidates is analyzed in a following section. Each candidate $h$ designs an electoral platform specifying the amount of the public good $g^{h}$ and the tax rate $t^{h}$. We assume that candidates can commit to the proposed platform, which will become the implemented policy if the candidate is elected. The no-commitment case will be considered later on. The cost of the public good, $c(g)$ is the same for each candidate, it is known by voters and it is characterized by $c(0)=0, c^{\prime}>0, c^{\prime \prime} \geq 0$.

The utility of voter $i$ associated with candidate $h$ 's platform is given by a component $v(\cdot)$ which depends on the policy parameters and which is common to all voters, and additional terms that capture voters' heterogeneity:

$$
U_{i}^{h}=v\left(t^{h}, g^{h}\right)+\epsilon_{i}^{h}+\beta^{h}=w\left(1-t^{h}\right)+a\left(g^{h}\right)+\epsilon_{i}^{h}+\beta^{h}
$$

where $w$ is the initial endowment over which the taxes are levied, and the utility from the public good is $a(g)$, with $a(0)=0, a^{\prime}>0, a^{\prime \prime}<0$. The heterogeneity of voters is given by the term $\epsilon_{i}^{h}$, which is specific to each voter with respect to each candidate, and the term $\beta^{h}$ which is the same for all voters with respect to each candidate, and corresponds to an aggregate bias common to the electorate, due for instance to personal attributes of the candidate, a better knowledge etc., including a (positive or negative) incumbent bias. The voters' preferences for candidate $h$, therefore, are heterogeneous around this mean $\beta^{h}$ according to the $\epsilon_{i}^{h}$ distribution ${ }^{6}$.

The terms $\epsilon_{i}^{h}$ are i.i.d and are drawn from some distribution with zero mean and finite variance. Let $F(x)$ be the continous symmetric cumulative distribution of the difference in the terms $\epsilon_{i}^{k}-\epsilon_{i}^{h}$, with $F(0)=1 / 2$. The term $\beta^{h}$ is drawn from some continuous atomless distribution with zero mean and finite variance.

\footnotetext{
${ }^{6}$ See Enelow and Hinich (1982) for a similar specification of voters' preferences. Borrowing from the product differentiation literature, and abusing a bit in terminology, the terms $\epsilon$ refer to horizontal heterogeneity in the evaluation of the platform among voters, while the $\beta$ term captures a vertical attribute of the candidate.
} 
The institutional setting is characterized by two features: majority voting and winner-takes-all. The former implies that the platform which obtains the majority of votes becomes the policy implemented; the latter specifies that the politician in office does not share the rents with the opponent.

The timing of the game is as follows. At $t=0$ Nature draws the $\epsilon$ 's and $\beta$ 's, which are observed by the voters; candidates' information on these elements will be specified in different ways later on. At $t=1$ the two candidates simultaneously specify their electoral platform. Finally, at $t=2$ the voters cast their vote given the candidates' electoral platforms. The candidate who obtains the majority of votes is elected and his platform is realized.

Voter $i$ chooses candidate $h$ if $U_{i}^{h}>U_{i}^{k}$ and flips a coin if $U_{i}^{h}=U_{i}^{k}$. In this set-up we consider two cases according to candidates' information. As we show in the next sections, what makes the difference in the electoral game is whether candidates know the distributions of the $(\epsilon+\beta)$ components or if they ignore their mean (and median), i.e. if they don't know the realized $\beta$ 's. We shall refer to this latter case as candidates' aggregate uncertainty.

\section{Model I: The candidates know the distribution of voters}

Consider the case in which the candidates know the realizations $\beta^{h}$ and $\beta^{k}$ but don't observe the $\epsilon_{i}^{h}$ and $\epsilon_{i}^{k}$ terms, knowing only their distribution. In this case the candidates cannot observe the utility of every single voter $i$, but they are able to reconstruct the distribution of the $\epsilon_{i}+\beta$ terms associated to each platform: the mean and median of this distribution is $\beta$. Voters' behaviour can therefore be anticipated in probabilistic terms. Voter $i$ will vote for $h$ if $U_{i}^{h}>U_{i}^{k}$, i.e.

$$
v\left(t^{h}, g^{h}\right)-v\left(t^{k}, g^{k}\right)+\beta^{h}-\beta^{k}>\epsilon_{i}^{k}-\epsilon_{i}^{h}
$$

where $v\left(t^{h}, g^{h}\right)$ is the (deterministic) utility, defined in equation (1), associated to $h$ 's platform and common to all voters. Given candidates' information, the probability that voter $i$ votes for candidate $h, \pi_{i}^{h}$, is therefore ${ }^{7}$ :

$$
\pi_{i}^{h}=F\left(v\left(t^{h}, g^{h}\right)-v\left(t^{k}, g^{k}\right)+\beta^{h}-\beta^{k}\right)
$$

\footnotetext{
${ }^{7}$ Since $F(x)$ is continous, the probability that $U_{i}^{h}=U_{i}^{k}$ has zero measure. Hence the probability that voter $i$ will choose candidate $h$ can be associated with the event $U_{i}^{h} \geq U_{i}^{k}$.
} 
Since $I$ is very large, the expected vote share for candidate $h, \Pi^{h}$, has the same expression. The majority rule then allows to compute the probability of candidate $h$ winning the elections, $G^{h}$ :

$$
G^{h}= \begin{cases}1 & \text { if } v\left(t^{h}, g^{h}\right)-v\left(t^{k}, g^{k}\right)>\beta^{k}-\beta^{h} \\ 1 / 2 & \text { if } v\left(t^{h}, g^{h}\right)-v\left(t^{k}, g^{k}\right)=\beta^{k}-\beta^{h} \\ 0 & \text { if } v\left(t^{h}, g^{h}\right)-v\left(t^{k}, g^{k}\right)<\beta^{k}-\beta^{h}\end{cases}
$$

Given the winner-takes-all rule after elections, the expected utility of candidate $h$ is

$$
U_{h}=G^{h}\left(I w t^{h}-c\left(g^{h}\right)\right)=G^{h} R^{h}
$$

where $R^{h}$ are the rents kept by candidate $h$. We can now proceed to analyze the equilibrium of the game.

We start our equilibrium analysis considering the case in which no candidate has a bias advantage, i.e. $\beta^{h}=\beta^{k}$.

Consider the locus $R_{\mathbf{0}}^{h}$ of all the policy combinations $\left(g^{h}, t^{h}\right)$ such that candidate $h$ obtains no rent: it is defined by the condition $c\left(g^{h}\right)=w I t^{h}$. It is easy to show that the locus $R_{0}^{h}$ passes through the origin in the $\left(t^{h}, g^{h}\right)$ space, it is increasing and concave. Since we assume that the cost of providing the public good is the same for both candidates, $R_{0}^{k}$ has exactly the same shape.

Now consider, for given rival's platform, the locus $G_{1 / 2}^{h}$ of all the policy combinations $\left(t^{h}, g^{h}\right)$ such that, for given rival platform $\left(t^{k}, g^{k}\right)$, the probability of winning is $1 / 2$ : this is defined implicitly by $v\left(t^{h}, g^{h}\right)-v\left(t^{k}, g^{k}\right)=$ $\beta^{k}-\beta^{h}$. Notice that $G_{1 / 2}^{h}$ identifies the median platforms. It can be easily established that the locus $G_{1 / 2}^{h}$ is increasing and convex. Moreover, when $\beta^{h}=\beta^{k}$ the loci $G_{1 / 2}^{h}$ and $G_{1 / 2}^{k}$ are identical. The following proposition establishes the existence and characterizes the equilibrium.

Proposition 1 If $\beta^{h}=\beta^{k}$ the candidates gain no rents in equilibrium $\left(R^{h}=R^{k}=0\right)$, they offer identical platforms characterized by the efficient provision of the public good and win the elections with probability $1 / 2$.

Proof: When $\beta^{h}=\beta^{k}$, by offering the same platform as the rival, each candidate can get the associated rents with probability $1 / 2$; that is, if candidate $k$ 's platform gives rents $R^{k}$, by replicating $k$ 's platform candidate $h$ can get $R^{h}=R^{k}$ and $G^{h}=1 / 2$. Hence in equilibrium candidate $h$ cannot get less than $R^{k} / 2$. Moreover, he cannot gain more than $R^{k} / 2$ in equilibrium: 
in this case $k$ would deviate offering $h$ 's platform. We conclude that in an equilibrium it must be that $R^{h}=R^{k}$ and $G^{h}=1 / 2$. However, if $R^{h}=R^{k}>$ 0 and $G^{h}=1 / 2$, each candidate can reduce taxes or increase the supply of the public good slightly and win the election with a platform that guarantees positive rents. Hence, a necessary condition for an equilibrium is $R^{h}=R^{k}=$ 0 and $G^{h}=1 / 2$. In other words, the two platforms must belong to $R_{0}^{h}, G_{1 / 2}^{h}$, $R_{0}^{k}$ and $G_{1 / 2}^{k}$. However, this is not sufficient for an equilibrium. Consider candidate $h$ : we know that $R_{0}^{h}$ and $G_{1 / 2}^{h}$ are increasing, the former is concave and passes through the origin, the latter is convex and shifts parallely with candidate $k$ 's platform. Hence, either $G_{1 / 2}^{h}$ is always above $R_{0}^{h}$, or they are tangent or there are two intersections between the two curves. In this latter case there is a subset of policy combinations between the two curves, above $G_{1 / 2}^{h}$ and below $R_{0}^{h}$, in which $R^{h}>0$ and $G^{h}>1 / 2$. If, given candidate $k$ 's platform, there are two intersections between $G_{1 / 2}^{h}$ and $R_{0}^{h}$, a platform $h$ on one of the intersection points cannot be an equilibrium even if the necessary condition above holds, since $h$ can profitably deviate choosing a platform in the subset between the two curves. Hence in equilibrium the two platforms must be at the tangency point between the two curves. Since $R_{0}^{h}$ and $R_{0}^{k}$ do not depend on rival's platform, the tangency point is symmetric and $t^{h}=t^{k}$ Notice that the slope of $G_{1 / 2}^{h}$ is $w / a^{\prime}\left[a^{-1}\left(a\left(g^{k}\right)+w\left(t^{h}-t^{k}\right)\right)\right]$ and the slope of $R_{0}^{h}$ is $w I / c^{\prime}\left[c^{-1}\left(w I t^{h}\right)\right]$, and the same for $k$ Since $t^{h}=t^{k}$ the former is $w / a^{\prime}\left(g^{h}\right)$ and since $R^{h}=0$ the latter is $w I / d^{\prime}\left(g^{h}\right)$. Equating the slopes we obtain for both candidates $c^{\prime}(g)=I a^{\prime}(g)$, and $g=g^{h}=g^{k}$, i.e. the usual Samuelson condition for the efficient provision of a public good. With identical platforms, $G^{h}=1 / 2$.

When the candidates know the distribution of voters' heterogeneity and there is no bias in favour of any candidate, electoral competition drives the political rents down to zero. The majority voting and the winner-takes-all rules make winning the election extremely appealing, and all the rents are dispersed in the attempt to obtain the office.

Since Downs' seminal contribution, the analysis of political competition in elections has been deeply influenced by the theory of imperfect competition, namely by the Hotelling approach to product differentiation. Hence, it seems natural to compare our result to an equivalent setting referred to oligopolistic markets. Indeed, the probabilistic voting model we have used in 
our set-up is very similar to a probabilistic model of product differentiation ${ }^{8}$. However, some interesting differences emerge between the two applications, which seem to suggest that applying a Hotelling-type approach to political competition allows to reach new and different results with respect to the original IO literature.

Suppose that two firms offer the same product but consumers' utility depends also on additional terms $\epsilon$ which are specific to each consumer with respect to each product, and not observed by firms. More precisely, assume that the utility of consumer $i$ purchasing product $h$ is $U_{i}^{h}=a-p^{h}+\epsilon_{i}^{h}$. To maintain the parallel with our electoral competition model, let $F(x)$ be the continuous and symmetric distribution in the difference of the terms $\epsilon_{i}^{h}-\epsilon_{i}^{k}$ with $F(0)=1 / 2$. In this case, the expected demand for product $h$ is $I F\left(p^{k}-p^{h}\right)$ where $I$ is the number of consumers. Hence, even with identical products, the expected demand for each product will be smooth, since by reducing the price a firm will expect to gain only a fraction of consumers, whose difference in the $\epsilon$ terms is lower than the difference in prices. Then, assuming zero marginal costs, the profits are $p^{h} I F\left(p^{k}-p^{h}\right)$, and it is easy to check that the Nash equilibrium in prices is $p^{h}=I / 2 F^{\prime}(0)$ : the more heterogeneous the consumers' evaluation, the lower $F^{\prime}(0)$ and the higher the equilibrium price. Hence, uncertainty on the heterogeneity of consumers is sufficient in an oligopoly model to relax price competition, avoiding the Bertrand result of complete rents dissipation.

In our probabilistic voting model of electoral competition, on the contrary, political rents are completely dissipated even if the candidates are uncertain on the voters' evaluations $\epsilon$. More precisely, the specific distribution $F(\cdot)$ of the $\epsilon$ terms plays no role in equilibrium. This is a striking difference between the two classes of models. When a candidate competes in elections under a majority voting and winner-takes-all rule, all that matters is to gain the majority of votes. Any symmetric distribution $F(x)$ with finite variance ${ }^{9}$ translates in the same discontinuous function of the probability of winning $G^{h}$ as (4), no matter how dispersed is the distribution in the difference of the $\epsilon$ terms. All that matters in our institutional framework is to gain the lead on the rival, but how large is the expected margin is irrelevant. In a sense, the institutional set-up that characterizes our model reproduces the standard discontinous payoff function of the Bertrand type

\footnotetext{
${ }^{8} \mathrm{And}$, in turn, there is a one-to-one relation between a probabilistic model of product differentiation and an address model à la Hotelling. See Anderson, De Palma and Thisse (1991).

${ }^{9} \mathrm{~A}$ fortiori, if the distribution of the $\epsilon$ terms is degenerate, this result holds.
} 
of competition even when some degree of heterogeneity occurs ${ }^{10}$.

We consider now the equilibrium outcomes when there exists a bias in favour of a candidate: without loss of generality let $\beta^{h}<\beta^{k}$, i.e. ceteris paribus candidate $k$ is preferred, on average, by all the voters. In the following Lemma we state the necessary conditions for an equilibrium: they can be approximated by an "almost optimal" strategy profile at which the candidates have the same probability of winning ${ }^{11}$. Given this allocation, the stronger candidate will then win the elections proposing a platform slightly more favourable to the voters, i.e. implementing a strategy profile in the neighborhood of the "almost optimal" point.

Lemma 2 When $\beta^{k}>\beta^{h}$, in equilibrium candidate $h$ platform is in the neighborhood of a point which belongs to the loci $R_{0}^{h}$ and $G_{1 / 2}^{h}$ and candidate $k$ platform is in the neighborhood of a point in the sets $R_{\alpha}^{k}$ and $G_{1 / 2}^{k}$, where $R_{\alpha}^{k}$ is defined by the condition $R^{k}=w I t^{k}-c\left(g^{k}\right)=\alpha$

Proof: When voters are biased in favour of candidate $k, k$ wins the elections. We approximate the equilibrium platforms by still using the locus $G_{1 / 2}^{h}: k$ will choose a platform slightly more favourable to the voters, and will win the elections. Hence, the equilibrium platforms will be in a neighborhood of the loci $G_{1 / 2}^{h}, G_{1 / 2}^{k}, R_{0}^{h}, R_{\alpha}^{k}$. The Bertrand type competition between the candidates will drive the rents down, until the weaker candidate $h$ is left with no rents. Hence $R^{h}=0$. Since candidate $k$ is relatively preferred by the voters, he can offer a less favourable platform and still win the elections. This platform lies in a neighborhood of the locus $G_{1 / 2}^{k}$. Since $h$ gains no rents and $k$ 's platform is less favourable to the voters, $R^{k}=\alpha>0$.

\footnotetext{
${ }^{10} \mathrm{~A}$ different result can be found in Grillo and Polo (1993): in a model similar to the present one, the winner-takes-all rule is substituted with a class of continuous sharing rules that map the share of votes collected into a share of political rents, even if the candidate looses the elections. Hence, the majority voting rule selects the policy implemented and the sharing rule determines how the associated rents are distributed to the politicians. In this set-up, the more dispersed are the voters, the higher the rents appropriated, in line with the product differentiation case.

${ }^{11}$ The approximation intends to capture the fact that the payoff functions have not a properly defined maximum, and the optimal policy can be approximated in a neighborhood of an "almost optimal" allocation. A more rigorous treatment would require to define the strategy space over a discrete set (of policy parameters), obtaining a well defined maximum. We omit to treat the problem in such a way since no additional insight is obtained.
} 
The necessary conditions for an equilibrium in the electoral game suggest that the bias in favour of a candidate allows him to gain positive rents. In the following proposition we characterize the equilibrium platforms, describing the features of the policy parameters in the neighborhood of which the winning and loosing electoral programs are designed.

Proposition 3 Candidate $k$ in equilibrium obtains positive rents. The equilibrium platforms are characterised by:
i) $R^{k}=\alpha=I\left(\beta^{k}-\beta^{h}\right)>0$
ii) $t^{k}=t^{h}+\left(\beta^{k}-\beta^{h}\right) / w$;
iii) $g^{h}=g^{k}$;
iv) $I a^{\prime}\left(g^{h}\right)=c^{\prime}\left(g^{h}\right)$;

Proof: The argument used in the case $\beta^{h}=\beta^{k}$ to establish that the equilibrium platform must be at the tangency point between the $R_{0}^{h}$ and the $G_{1 / 2}^{h}$ loci still holds. Moreover, among the policy combinations on $G^{k}=1 / 2$, candidate $k$ will choose the one associated with the highest rents $\alpha$, at the tangency point between $G_{1 / 2}^{k}$ and $R_{\alpha}^{k}$. Notice that along the $G_{1 / 2}^{h}$ locus $g^{h}=a^{-1}\left[a\left(g^{k}\right)+w\left(t^{h}-t^{k}\right)+\left(\beta^{k}-\beta^{h}\right)\right]$ and an equivalent expression for $g^{k}$. Hence, we can write the tangency conditions as:

$$
c^{\prime}\left(g^{h}\right)=I a^{\prime}\left[a^{-1}\left(a\left(g^{k}\right)+w\left(t^{h}-t^{k}\right)+\left(\beta^{k}-\beta^{h}\right)\right)\right]
$$

for candidate $h$ and

$$
c^{\prime}\left(g^{k}\right)=I a^{\prime}\left[a^{-1}\left(a\left(g^{h}\right)-w\left(t^{h}-t^{k}\right)-\left(\beta^{k}-\beta^{h}\right)\right)\right]
$$

for candidate $k$. Now suppose $g^{h} \geq g^{k}$. Since $c^{\prime \prime} \geq 0, c^{\prime}\left(g^{h}\right) \geq c^{\prime}\left(g^{k}\right)$. Hence

$$
a^{\prime}\left[a^{-1}\left(a\left(g^{k}\right)+w\left(t^{h}-t^{k}\right)+\left(\beta^{k}-\beta^{h}\right)\right)\right] \geq a^{\prime}\left[a^{-1}\left(a\left(g^{h}\right)-w\left(t^{h}-t^{k}\right)-\left(\beta^{k}-\beta^{h}\right)\right)\right]
$$

and, being $a^{\prime \prime}<0$,

$$
a\left(g^{h}\right) \geq a\left(g^{k}\right)+2\left(w\left(t^{h}-t^{k}\right)+\left(\beta^{k}-\beta^{h}\right)\right)
$$

Since in equilibrium $G^{h}=1 / 2$,

$$
a\left(g^{h}\right)=a\left(g^{k}\right)+w\left(t^{h}-t^{k}\right)+\left(\beta^{k}-\beta^{h}\right)
$$

Putting toghether: 


$$
w\left(t^{h}-t^{k}\right)+\left(\beta^{k}-\beta^{h}\right) \leq 0
$$

that implies $g^{h} \leq g^{k}$. Hence, to avoid a contradiction $g^{h}=g^{k}$ and $w\left(t^{h}-\right.$ $\left.t^{k}\right)+\left(\beta^{k}-\beta^{h}\right)=0$. As a consequence $t^{h}=t^{k}-\left(\beta^{k}-\beta^{h}\right) / w$ and $c^{\prime}\left(g^{h}\right)=$ $I a^{\prime}\left[a^{-1}\left(a\left(g^{h}\right)\right)\right]=I a^{\prime}\left(g^{h}\right)$, i.e. the two platforms offer the public good at the efficient level. Finally, substituting in $R^{k}=w I t^{k}-c\left(g^{k}\right)$ for the equilibrium tax rate and using $g^{k}=g^{h}$ we get $R^{k}=w I\left(t^{h}+\left(\beta^{k}-\beta^{h}\right) / w\right)-c\left(g^{h}\right)=$ $R^{h}+I\left(\beta^{k}-\beta^{h}\right)=\alpha$ being $R^{h}=0$.

Voter's bias allows the favoured candidate to gain rents in equilibrium. Even though competition is intense in this environment, rents are not completely dissipated, due to the candidate's advantage which cannot be eliminated: in this sense voters' bias determines the emergence of "Ricardian" or classical rents. Moreover, notice that the equilibrium platforms when $\beta^{k}>\beta^{h}$ converge to the ones when voters' preferences are unbiased as $\beta^{k} \rightarrow \beta^{h}$. With quasi-linear utilities the efficient provision of the public good still holds and all the rents are obtained through an increase in the tax rate: it is in fact convenient to set the public good at the level which maximizes social welfare, increasing the share of surplus appropriated through a higher tax rate. We shall consider later on whether, with non separable utility, the distortion in the tax rate goes along with a distortion in the supply of the public good.

We move now to the case when candidates have aggregate uncertainty.

\section{Model II: The candidates don't observe the mean of the distribution}

We have seen in the previous section that if the candidates know the relevant parameters of the distribution of voters' heterogeneity - mean and variance in our symmetric setting - electoral competition induces the dispersion of political rents: positive rents are preserved only if there exists a positive bias in favour of a candidate. We consider now the case in which the candidates do not observe the mean (and median) of voters' distribution, i.e. a situation in which the candidates are uncertain on the realization of the $\beta$ terms. In this case the probability of winning the elections is no longer a discontinuous step function.

More precisely, we assume that while the two terms are not observed, the two candidates know the distribution of the difference $\beta^{k}-\beta^{h}$, which 
can be interpreted as the net bias in favour of candidate $k$. Let $H(x)$ be the associated cumulative distribution function: we assume it to be symmetric around zero and continuous, with $H(x)=1-H(-x)$, convex for $x \leq 0$ and concave for $x \geq 0$. Moreover, we assume that when the distribution of $\beta^{k}-\beta^{h}$ is more dispersed, this function becomes flatter at $x=0$, a property shared by most of the symmetric distributions as the normal or the logistic functions. Hence the derivative $H^{\prime}(0)$ is negatively linked to the variance of the distribution, and in the limit tends to infinity as the distribution becomes degenerate.

Candidates know that $h$ will win the elections if $\left.v\left(t^{h}, g^{h}\right)-v\left(t^{k}, g^{k}\right)\right)>$ $\beta^{k}-\beta^{h}$. However, since the last terms are not observed they can only asses the probability of $h$ winning the elections, $G^{h}$, as:

$$
G^{h}=H\left(v\left(t^{h}, g^{h}\right)-v\left(t^{k}, g^{k}\right)\right)
$$

Candidate $h$ utility is then, as before, $U_{h}=G^{h}\left(I w t^{h}-c\left(g^{h}\right)\right)=G^{h} R^{h}$, but it is now a smooth function. Before proceeding with the equilibrium analysis, let us define $\bar{R}=w I-c\left(g^{h}\right)$ as the maximum surplus that a candidate can appropriate when offering the public good at the efficient level and setting a tax rate $t^{h}=1$.

The equilibrium in the electoral game is analyzed in the following proposition.

Proposition 4 The equilibrium rents $R^{h}$ lie in the interval $[0, \bar{R}]$; they are increasing in the variance of the distribution of voters' bias, and tend to zero as the variance tends to zero. In equilibrium the candidates offer identical platforms characterized by the efficient provision of the public good, and win the elections with probability $1 / 2$.

Proof: If the constraint on the tax rate $t^{h} \leq 1$ does not bind, the first order condition for a Nash equilibrium are:

$$
\begin{gathered}
\frac{\partial U_{h}}{\partial t^{h}}=\frac{\partial H}{\partial t^{h}} R^{h}+w I H=-w H^{\prime} R^{h}+w I H=0 \\
\frac{\partial U_{h}}{\partial g^{h}}=\frac{\partial H}{\partial g^{h}} R^{h}-c^{\prime} H=a^{\prime} H^{\prime} R^{h}-c^{\prime} H=0
\end{gathered}
$$

At a symmetric equilibrium, the former implies $R^{h}=I / 2 H^{\prime}(0)$ which is positive and increases as the variance of the distribution increases, reducing $H^{\prime}(0)$. Substituting in the latter derivative we obtain $H(0)\left(a^{\prime} I-c^{\prime}\right)=0$ 
which is the usual Samuelson condition for the efficient provision of a public good. The second partial derivatives are:

$$
\begin{array}{r}
\frac{\partial^{2} U_{h}}{\partial t^{h 2}}=w^{2} I A<0 \\
\frac{\partial^{2} U_{h}}{\partial g^{h 2}}=\left(a^{\prime \prime} I-c^{\prime \prime}\right) H+a^{\prime} c^{\prime} A<0
\end{array}
$$

where $A=\left(H^{\prime \prime} H I / H^{\prime}-2 I H^{\prime}\right)<0$ and the determinant of the Hessian matrix is $w^{2} I A\left(a^{\prime \prime} I-c^{\prime \prime}\right) H>0$. Hence the second order conditions hold. When the variance increases more and more (and $H^{\prime}(0)$ falls consequently, we reach a point at which $R^{h}=\bar{R}$; the feasibility constraint on the tax rate becomes binding and the same corner solution occurs for even more dispersed distributions.

The intuition for the result just proved can be explained as follows: when the bias becomes more dispersed, offering a platform marginally more favourable to the voters increases only slightly the probability of winning, while it reduces the rents kept if elected: as a result, the incentive to compete strongly with the rival is reduced, and in equilibrium the political rents increase.

This result may seem surprising, since we are assuming that the distribution in voters' bias $\beta^{k}-\beta^{h}$ is symmetric around zero. That means that the candidates expect a bias in favour of either of them to occur, according to the distribution $H(\cdot)$. The crucial point is that while a positive or negative bias occur with the same probability, they are not equivalent and do not cancel out in terms of final payoffs: with a positive bias the candidate may win the elections even with a platform entailing positive rents, while with a negative realization he looses and gets nothing. In other words, the expected payoffs of a candidate depend only on the positive realizations of the bias in favour of him. A platform less favourable to the voters requires a higher (positive) bias to win the elections. Since those (positive) realizations occur with a higher probability when the distribution of $\beta^{k}-\beta^{h}$ becomes more dispersed, while the corresponding negative biases pay always zero, the marginal incentive to offer an electoral platform with large political rents increases.

There is a natural interpretation of candidates' uncertainty in our model. Candidates face a heterogeneous electorate according to the $\epsilon$ terms. If they observe the $\beta$ 's, they know the mean of this distribution and are able to 
compute the platform associated with $G^{h}=1 / 2$, i.e. the median platform. If the $\beta$ 's are not observed, the candidates don't know the mean of the voters' distribution, i.e they are uncertain on which is the median platform.

We feel that this kind of aggregate uncertainty is quite natural and realistic in political competition, and makes the result obtained rather general and disturbing: even adopting a competition-inducing institutional framework, as a majority plus winner-takes-all rule, we are unable to obtain complete rent dissipation when there is aggregate uncertainty on voters' behaviour.

We have considered so far a symmetric distribution of voters' bias around zero, implying that no candidate has a net positive advantage in expected terms. It is interesting to extend the model to the case in which, although uncertain abount the real attitude of the electorate, the candidates expect one of them to have a lead in the campain. Let $x$ be the net expected bias for candidate $h$, which can be positive or negative. Then, $h$ expects to win the elections if $v\left(t^{h}, g^{h}\right)-v\left(t^{k}, g^{k}\right)+x \geq \beta^{k}-\beta^{h}$. The distribution of $\beta^{k}-\beta^{h}$ is assumed, as before, to be symmtrically distributed around 0 , with cumulative distribution $H(\cdot)$. The probability of winning the election for candidate $h$ is therefore

$$
G^{h}=H\left(v\left(t^{h}, g^{h}\right)-v\left(t^{k}, g^{k}\right)+x\right)
$$

In the following proposition we establish the existence and characterize the equilibrium in case of an expected bias in favour of a candidate.

Proposition 5 When there is an expected bias in favour of one of the candidates, i.e. $x \neq 0$, an equilibrium exists provided that the two platform do not diverge too much. The favourite candidate chooses a platform associated with higher rents and wins the elections with a higher probability.

Proof: We first characterize the equilibria and then move back to the issue of existence. The equilibrium conditions can be obtained as in the previous proposition. From the first order conditions we obtain the expressions:

$$
\begin{array}{r}
R^{h}=\frac{I H\left(v\left(t^{h}, g^{h}\right)-v\left(t^{k}, g^{k}\right)+x\right)}{H^{\prime}\left(v\left(t^{h}, g^{h}\right)-v\left(t^{k}, g^{k}\right)+x\right)} \\
H(\cdot)\left(a^{\prime} I-c^{\prime}\right)=0
\end{array}
$$


The latter equation implies $g^{h}=g^{k}$ at the efficient level. Consider the case $v\left(t^{h}, g^{h}\right)-v\left(t^{k}, g^{k}\right)+x>v\left(t^{k}, g^{k}\right), v\left(t^{h}, g^{h}\right)-x$. It immediately follows that $G^{h}>G^{k}$. Moreover, since $H(\cdot)$ is symmetric, $H^{\prime}\left(v\left(t^{h}, g^{h}\right)-v\left(t^{k}, g^{k}\right)+x\right)=$ $H^{\prime}\left(v\left(t^{k}, g^{k}\right)-v\left(t^{h}, g^{h}\right)-x\right)$ and $R^{h}>R^{k}$. Since $g^{h}=g^{k}, t^{h}>t^{k}$. The above inequality can be rewritten as $t^{h}<t^{k}+x / w$; putting toghether the two restrictions on the tax rate, we conclude that $x>0$, i.e. that $h$ is the favourite candidate. Hence, when $x>0, R^{h}>R^{k}$ and $G^{h}>1 / 2$.

Consider then the issue of existence. The second order conditions have the same general expression considered in the previous proposition. But now the candidate with a negative expected bias offers a platform in the region where $H$ is convex, and therefore $H^{\prime \prime}>0$. Since we need $\left(H^{\prime \prime} H / H^{\prime}-2 H^{\prime}\right)<$ $0, H^{\prime \prime}$ cannot be too large, i.e. the two platforms cannot diverge too much. $H^{\prime \prime}$ depends on the variance of the distribution and on the divergence of the platforms, which is endogenous; hence, whether the second order conditions are met depends on the bias $x$ and on the specific distribution $H(\cdot)^{12}$.

The result obtained with a positive expected bias tells us that gaining the lead in a campain relaxes even more electoral competition. Moreover, this result suggests an interesting effect of incumbency: if the incumbent is more appreciated, on average, by the electorate, due for instance to a better knowledge, he has an advantage in the campain and wins the election with a higher probability and higher rents. Hence, we might observe a relative persistence of the politicians and an increasing distortion of the policies over time.

So far we have obtained that in any equilibrium the public good is offered at the efficient level which equates the sum of the marginal rates of substitution and the marginal cost. This result depends entirely on the assumption of quasi-linearity of voters' preferences. We briefly consider if, with non separable utility, an increase in taxes goes along with a distortion in the provision of the public good. Assume that $v\left(y, g^{h}\right)$ is no more separable in private consumption $y=w\left(1-t^{h}\right)$ and the public good, with positive and decreasing marginal utilities $v_{y}$ and $v_{g}$. The probability of winning the election for candidate $h$ follows the usual expression. We can now easily prove the following result.

\footnotetext{
${ }^{12}$ It can be shown that, if $H(\cdot)$ is a logistic distribution, the second order conditions always hold, i.e. for any $x$ and for given variance, the equilibrium is always associated with a degree of divergence consistent with the existence of the equilibrium. See proposition 8 for a proof.
} 
Proposition 6 When voters' preferences are not separable in money (private consumption) and the public good, positive political rents are associated with overtaxation and underprovision of the public good.

Proof: Following the lines of the previous proposition's proof, from the first order conditions in a symmetric equilibrium we obtain

$$
\begin{gathered}
R^{h}=\frac{I H(0)}{H^{\prime} v_{y}} \\
I v_{g} / v_{y}=c^{\prime}
\end{gathered}
$$

The second condition always holds, but now the marginal utility of money is no more constant and the emergence of rents may cause a distortion in the supply of the public good. With positive rents we have a variation in both the tax rate and the supply of the public good with respect to the balanced budget benchmark. First we show that the adjustments in $t^{h}$ and $g^{h}$ must be in opposite directions. Suppose that with positive rents $t^{h}$ increases, reducing private consumption and increasing the marginal utility $v_{y}$; if also $g^{h}$ increases, the marginal cost $c^{\prime}$ is higher and the marginal rate of substitution must increase as well. Since the numerator $v_{y}$ is higher, we need an even larger increase in $v_{g}$, which is consistent only with a fall in $g^{h}$, a contradiction. Applying the same argument we can show also that a contemporaneous fall in the tax rate and the public good is inconsistent with the equilibrium conditions. We are left with two possible adjustments, an increase in the tax rate coupled with a fall in $g^{h}$ or the opposite: both are consistent with the argument we applied above, but only the former case allow the candidate to obtain higher rents in equilibrium. Hence, when political rents are positive in equilibrium, they are associated with an increase in taxes and a contraction in the public good supplied.

The proposition just proved allows to evaluate all the consequences of imperfect electoral competition due to candidates' imperfect information: positive rents arise in equilibrium through an increase in taxation and a contraction in the public good provided.

\section{Extensions}

Once discussed our main result, we explore some extensions of the model. First, we add an ideological variable in the platforms, allowing the candidates to partially influence also the idiosyncratic component of voters' 
preferences. Then we consider the effect on equilibrium rents of an increase in the numer of candidates. Finally, we drop the assumption of perfect commitment to the annouced platforms, analyzing a second source of political rents, associated with ex-post moral hazard of the elected candidate.

\subsection{Ideological platforms}

In the basic model, the proposed platforms influence all the voters in the same way through the common component $v\left(t^{h}, g^{h}\right)$. The overall utility differs across voters through the terms $\epsilon_{i}^{h}+\beta^{h}$, but this heterogeneous effect can not be influenced by the candidate and is exogeneous with respect to the policy variables. As a consequence, although uncertainty is crucial in the working of electoral competition, the unobserved component is completely out of the control of the candidates. In this section we remove this assumption, exploring the case in which candidates are able to choose the differentiated effects of their platforms across voters.

In order to maintain the model as similar as possible to the benchmark case, we assume that the component $\epsilon_{i}^{h}$ is referred to an ideological dimension, over which the candidates can take a position. Hence, a platform is now a triple $\left(t^{h}, g^{h}, x^{h}\right)$, what we call an ideological platform.

Let $i \in \Re$ be the spectrum of ideological positions of the electorate, with continuous cumulative distribution $F(\cdot)$ symmetric around 0 , and $x^{h} \leq 0 \leq$ $x^{k}$ the ideological positions taken by the two candidates. Notice that the median voter over the ideological dimension is located at $i=0$. Voter $i$ 's utility associated to candidate $h$ 's platform is:

$$
U_{i}^{h}=v\left(t^{h}, g^{h}\right)+\beta^{h}-\left(i-x^{h}\right)^{2}=w\left(1-t^{h}\right)+a\left(g^{h}\right)+\beta^{h}-\left(i-x^{h}\right)^{2}=
$$

where the quadratic expression corresponds to an increasing marginal disutility of voting a candidate far from the ideological positions of the voter. It is easy to see that, given the platforms of the candidates and the realizations of the $\beta$ 's, the expected vote share of candidate $h$, which includes all the voters to the left of the marginal voter $\hat{i}$, is:

$$
\Pi^{h}=F(\hat{i})=F\left(\frac{v\left(t^{h}, g^{h}\right)-v\left(t^{k}, g^{k}\right)+\beta^{h}-\beta^{k}+x^{k 2}-x^{h 2}}{2\left(x^{k}-x^{h}\right)}\right)
$$

When the candidates do not observe the realization of the $\beta$ 's, the expected vote share becomes a random variable, exactly as in the model already 
analyzed, and the same holds true for the probability of winning the election $G^{h}:$

$$
G^{h}=H\left(v\left(t^{h}, g^{h}\right)-v\left(t^{k}, g^{k}\right)+x^{k 2}-x^{h 2}\right)
$$

Notice that the probability of winning increases, for given platform, as the candidate moves toward the median voter $i=0$. Moreover, the model with ideological platforms is similar to the asymmetric bias case considered in the previous section, although now the bias can be influenced by the candidates. We can state a preliminary result which illustrates the effects of candidates' ideological positioning.

Lemma 7 Candidates' divergence $x^{k 2}-x^{h 2}$ over the ideological spectrum is irrelevant in equilibrium if they offer symmetric platforms, i.e. if $\left|x^{h}\right|=\mid$ $x^{k} \mid$.

Proof: It immediatly follows from the inspection of $G^{h}$.

We find here another time a relevant difference between a policy game and an analogous model referred to product differentiation. If firms offer different varieties, the mobility of consumers decreases when the degree of differentiation is higher, even with symmetric "locations", and the equilibrium prices go up. In a policy game, on the contrary, different degrees of symmetric "locations" over the ideological spectrum, although reducing voters' mobility, give rise to the same equilibria, corresponding to the one analized in the previous section where ideology was not part of the platforms. The intuition of this result can be found in the fact that, with majority voting and winner-takes-all, the magnitude of the marginal increase in votes induced by a more favourable platform, which is influenced by the ideological differentiation of the candidates, plays no role.

In order to simplify our analysis, and to offer an example in which the equilibrium always exists, we choose a specific form for the function $H(\cdot)$ : if the $\beta$ 's follow the double exponential distribution, the distribution of $\beta^{k}-\beta^{h}$ is logistic:

$$
G^{h}=H(\cdot)=\frac{\exp \left(\left(a\left(g^{h}\right)-w t^{h}-x^{h 2}\right) / \mu\right)}{\left.\exp \left(\left(a\left(g^{h}\right)-w t^{h}-x^{h 2}\right) / \mu\right)+\exp \left(a\left(g^{k}\right)-w t^{k}-x^{k 2}\right) / \mu\right)}
$$

where $\mu$ is a parameter posivitely correlated with the variance of the distribution. Notice that $\partial H / \partial t^{h}=(H(1-H) / \mu) \partial v / \partial t^{h}$ and similar expressions hold for $g^{h}$. 
The candidates define their electoral platform in a two stage sequence, which is intended to capture the idea that choosing an ideological characterization is a long term decision difficult to modify, while designing the tax rate and the supply of the public good is more contingent and short term in nature. Hence, the timing of the game is modified as follows. At $t=0$ Nature draws the $i$ 's and $\beta$ 's, which are observed by the voters but not by the candidates; At $t=1$ the two candidates simultaneously specify their ideological position $x^{h}$ and $x^{k}$. At $t=2$, once observed the $x$ 's, the candidates set the tax rate and the supply of the public good. Finally, the voters cast their vote given the candidates' electoral platforms. The candidate who obtains the majority of votes is elected and his platform is realized.

We can now consider the subgame perfect equilibrium in the game with ideological platforms.

Proposition 8 In equilibrium both candidates converge to the ideological position of the median voter. The political rents are identical to those $o b$ tained in Proposition 4.

Proof: Consider first the Nash equilibria in the subgames identified by a pair of ideological positions. Define $x=x^{k}-x^{h}$. Then we can apply all the results proved in Proposition 5 to the present case. Moreover, when $H(\cdot)$ is a logistic, the second partials ${ }^{13}$ are:

$$
\begin{aligned}
& \frac{\partial^{2} U_{h}}{\partial t^{h 2}}=-H w^{2} I / \mu<0 \\
& \frac{\partial^{2} U_{h}}{\partial g^{h 2}}=-H\left(c^{\prime \prime}-a^{\prime \prime} I+a^{\prime} c^{\prime} / \mu\right)<0 \\
& \frac{\partial^{2} U_{h}}{\partial g^{h} \partial t^{h}}=-H w a^{\prime} I / \mu<0
\end{aligned}
$$

which ensure that the second order conditions always hold. We know from Proposition 5 that the candidate with a positive bias obtains higher rents and a higher probability of winning in equilibrium. In our model, a positive bias can be obtained moving toward the median voter $i=0$. Hence, in the first stage game both candidates converge to this median ideological position.

\footnotetext{
${ }^{13}$ Remember that $H^{\prime}=H(1-H) / \mu$ and that $R^{h}=I \mu /(1-H)$.
} 
In this section we have analyzed the case in which the candidates' platforms entail both an ideological issue over which the voters are heterogeneous, and policy parameters that influence in the same way all the voters. We have seen that each candidate finds it convenient to choose a more central ideological position which pleases a majority of voters; the expected lead that is gained allows to obtain higher rents in equilibrium. Hence, the two dimensions of the platform are complementary one to each other. Although an interaction occurs between ideological positioning and rent extraction, the equilibrium allocation replicates the outcomes that can be obtained separately in a model with no political rents (convergence to the median voter) or in a model with no ideological issue (positive rents).

It is important to stress that the ideological characterization is not chosen in order to segment the electorate and to reduce the mobility of a subset of voters, that would require the two candidates to diverge. Instead, the platforms are designed with the aim of gaining an expected lead in the electorate over the rival, that can be obtained moving towards the center.

Finally, it is worth noting that the ideological choice of the candidate introduces in the game a reditributive issue, since by positioning a candidate is able to increase the utility of some voter while making others worse off. In our example, therefore, the conflict of interest between the electorate and the politicians (political rents), and that among voters (ideological positioning) interact.

\subsection{Electoral competition among $n$ candidates}

A possible way to reduce political rents might be to enhance competition in the political arena by increasing the number of candidates: since uncertainty relaxes the Bertrand-type competition, we want to check whether increasing competition by enlarging the number of competitors might solve our problem.

Under the same voting rule as before, $n$ candidates propose a platform; the candidate who obtains the relative majority of votes wins the office and realizes the proposed policy, keeping the political rents according to his electoral platform. Candidate $h$ will win the elections if $v\left(t^{h}, g^{h}\right)-v\left(t^{j}, g^{j}\right)>$ $\beta^{j}-\beta^{h}$ for $j=1, . ., n, j \neq h$. Since candidates do not observe the realizations of the $\beta$ s but know only the distribution of their difference, which is now $n-1$ dimensional, the probability that $h$ wins the election is: 


$$
\operatorname{Pr}\left(\beta^{1}-\beta^{h} \leq v\left(t^{h}, g^{h}\right)-v\left(t^{1}, g^{1}\right), \ldots, \beta^{n}-\beta^{h} \leq v\left(t^{h}, g^{h}\right)-v\left(t^{n}, g^{n}\right)\right)=G^{h}
$$

We generalize the assumptions used in the two candidate case by requiring the distribution $H\left(v\left(t^{h}, g^{h}\right)-v\left(t^{1}, g^{1}\right), \ldots, v\left(t^{h}, g^{h}\right)-v\left(t^{n}, g^{n}\right)\right)$ to be continuous, symmetric and differentiable; moreover $\partial^{2} H / \partial v^{2}>(<) 0$ if $H(\cdot)<(>) 1 / 2$ for each candidate $h$. Since symmetric platforms will occur in equilibrium, we have to carefully consider the properties of $H(0, \ldots, 0)$ or, in short, $H(0)$. First, we assume that the distribution of candidates' bias remains symmetric, with no incumbency effect, implying $H(0)=1 / n$ 14. It comes out that in equilibrium the crucial property of the function $H(\cdot)$ is the behaviour of $H(0) / \partial H(0) / \partial v^{h}=1 /\left(n \partial H(0) / \partial v^{h}\right)$. If the marginal probability of winning decreases in $n$ less rapidly than $H(0)$ does, the ratio above decreases as the number of candidates increases. Intuitively, if the distribution is such that the density is relatively concentrated and has little weight in the tails, the fall in the marginal probability is slower than that in the probability itself. We can now easily prove the following proposition.

Proposition 9 When $n$ candidates compete in the election, the equilibrium rents fall in $n$ if $H(0) / \partial H(0) / \partial v^{h}$ decreases in $n$. If $\lim _{n \rightarrow \infty} H(0) / \partial H(0) / \partial v^{h}=$ 0 the political rents are completely dissipated in the limit.

Proof: Consider the first order conditions at a symmetric allocation:

$$
\begin{gathered}
\frac{\partial U_{h}}{\partial t^{h}}=\frac{\partial H(0)}{\partial v^{h}} \frac{v^{h}}{\partial t^{h}} R^{h}+w I H(0)=-\frac{\partial H(0)}{\partial v^{h}} w R^{h}+w I H(0)=0 \\
\frac{\partial U_{h}}{\partial g^{h}}=\frac{\partial H(0)}{\partial v^{h}} \frac{v^{h}}{\partial g^{h}} R^{h}-c^{\prime} H(0)=\frac{\partial H(0)}{\partial v^{h}} a^{\prime} R^{h}-c^{\prime} H(0)=0
\end{gathered}
$$

we have therefore

$$
R^{h}=\frac{I H(0)}{\partial H(0) \partial v^{h}}
$$

which depends on the behaviour of $H(0) / \partial H(0) / \partial v^{h}$; substituting $R^{h}$, in the second equation, $I a^{\prime}=d^{d}$ which identifies the efficient provision of the public good. The second order conditions can be checked in the same way

\footnotetext{
${ }^{14}$ Examples of this function are the multinomial logit and probit, obtained when the individual $\beta$ 's are distributed according to the double exponential or normal distribution.
} 
as in the previous proposition. If $H(0) / \partial H(0) / \partial v^{h}$ goes to zero in the limit, rents are completely dissipated in the limit, while if that ratio tends to a constant, positive rents are kept even when infinite candidates compete.

The proposition above suggests that, depending on the features of the distribution $H(\cdot)$, increasing the number of candidates may reduce, and in the limit completely dissipate, political rents. For instance, if the individual $\beta$ 's are normally distributed, the probability of winning follows the probit model and $H(0) / \partial H(0) / \partial v^{h}$ decreases in $n$ and goes to zero in the limit, implying complete rent dissipation. On the contrary, if the $\beta$ 's are distributed according to the double exponential distribution, $H(\cdot)$ follows the multinomial logit and $H(0) / \partial H(0) / \partial v^{h}$ falls as $n$ increases but tends to a constant which is positively related to the variance in the distribution, i.e. the more dispersed is the distribution of the biases, the higher the rents even in the limit $^{15}$.

The intuition of the result above can be given along the following lines. Considering the first order conditions at a symmetric equilibrium, two effects are at work: offering a policy less favourable to voters reduces the probability of success for given rents and increases the rents for given probability. The magnitude of the first effect depends on $\partial H(0) / \partial v^{h}$ while that of the second depends on $H(0)$. If this latter term falls more than the former as $n$ increases, the incentives to offer a platform associated with high rents are reduced. If the relative effects on the two terms continues in the limit, complete dispersion occurs; otherwise, the two effects balance out in the limit and positive rents persist.

We would like to conclude this section suggesting that, if there are entry costs to participate in an electoral campain, the declining pattern of political rents just shown offers a possible way of determining the actual number of candidates through a condition of zero net expected rents.

\subsection{The no-commitment case}

So far we have maintained the assumption that the candidates are able to commit to the proposed policy once elected. This assumption, which is usually done in the literature on voting games, allows to concentrate the analysis on the stage of electoral competition. However, as pointed out at first by Ferejohn (1986), it cannot be taken too seriously, since it is difficult to imagine which device could be used to obtain such a commitment.

${ }^{15}$ See on this point Anderson et al. (1991), p.188. 
Once abandoned this assumption, there are many different ways in which candidates' imperfect commitment can be analyzed.

Ferejohn (1986) assumes that voters base their behaviour, and in particular their re-election decision, looking at the performance of the elected politician, with no concern on electoral promises: the voters set a threshold level of utility; if the politician in office guarantees at least that utility, they re-elect him, otherwise they patronize the challenger. Ferejohn's approach has striking consequences on the way we look at the interaction between voters and politicians: electoral competition and platforms are irrelevant, and political rents are uniquely determined by the incentive compatibility constraint of the politician, which has to be induced to guarantee the reservation utility of the voters rather than exploit them renoucing to be re-elected.

Although Ferejohn (1986) contribution is extremely important, we feel that his approach goes too far at the opposite extreme with respect to the perfect commitment case. In this latter, the candidates can fully commit to the promised platforms, and ex-post moral hazard is irrelevant. In the former, voters are able to perfectly commit to a threshold re-election rule, and ex-ante electoral competition becomes irrelevant. Our feeling, on the contrary, is that both electoral campaining and ex-post moral hazard are important pieces of the story. A full treatement of this issue is beyond the aim of this paper and is left to future research. In this section we want simply to explore another way of modeling voters' behaviour that may allow to give a role to both moral hazard and electoral competition in the determination of political rents.

We assume that the voters expect the candidates to implement the poposed policy once in office only if it is convenient to them, given voters' behaviour: voters' never vote ${ }^{16}$ for a candidate which has cheated in past elections or that is expected to cheat once in office. If the proposed platform guarantees to candidate $h$ a level of utility lower than a threshold $\hat{R}$ (that will be identified below) the voters expect that the candidate will not implement the platform, choosing instead to exploit his position implementing a less favourable policy. If the platform gives $h$ rents above $\hat{R}$, voters expect such a policy to be implemented and vote for $h$ if the utility associated to the rival's platform is lower, as considered in the commitment case $^{17}$.

\footnotetext{
${ }^{16}$ In order to rationalize this behaviour we have to set some restrictions on the support of the $\epsilon$ and $\beta$ terms.

${ }^{17}$ In order to avoid that the both candidates corner the voters, offering a platform that
} 
It is now easy to find the threshold level of rents $\hat{R}$. Consider infinitely living agents and let $V_{h}^{I}$ be the value of the game if candidate $h$ implements the policy once in office. It is $V_{h}^{I}=R^{h}+(\delta / n) V_{h}^{I}$, where $\delta$ is the discount factor and $1 / n$ the equilibrium probability of winning the next election. If candidate $h$, once in office, decides to exploit the voters and renouces to be re-elected, the value of the game when cheating is $V_{h}^{C}=\tilde{R} . \tilde{R}$, which is a parameter in our analysis is the highest level of rents allowed by the discretionary power of the politician in office. It is worth noting that this term is related to the constraints that the institutional setting imposes on the power of the elected politician, and therefore on the checks and balances that can limitate his ability to exploit the voters ${ }^{18}$

The elected candidate will choose to implement his platform if $V_{h}^{I} \geq V_{h}^{C}$, or

$$
R^{h} \geq \frac{n-\delta}{n} \tilde{R}=\hat{R}
$$

Since no voter would vote for a candidate offering a platform with rents lower than $\hat{R}$, candidate $h$ utility is $U_{h}=R^{h} G^{h}$ if $R^{h} \geq \hat{R}$ and 0 otherwise. We can now easily prove the following result.

Proposition 10 When candidates cannot commit to the proposed platform, under the voting rule described above the political rents are $\max \left\{R^{h}, \hat{R}\right\}$. When the number of candidates increases the political rents may initially decrease but then increase again up to $\tilde{R}$ in the limit.

Proof: Suppose that the equilibrium rents identified in the commitment case are higher than $\hat{R}$ : in this case the ex-post incentive compatibility constraint does not bind and the elected candidate finds it convenient to realize the promised policy and compete in the next election. If $R^{h}<\hat{R}$ the equilibrium strategy is given by the corner solution $\hat{R}$. We know from the previous proposition that $R^{h}$ is decreasing in $n$; moreover, $\hat{R}$ is increasing

cannot be trusted, we assume that in this case no voter goes to the polls and no candidate is elected.

${ }^{18}$ See on this point Persson, Roland and Tabellini (1997). It is beyond the aim of this paper to further analyze the determinants of $\tilde{R}$. However, we think that one interesting way of exploring the relations between pre-election and post-election constraints to the behaviour of politicians can be found along this direction. Abandoning the full commitment assumption, the post-election constraints allow to identify the politicians incentive compatibility constraints and the threshold rents that voters can use to define their voting rules. 
in $n$ and tends to $\tilde{R}$ as $n$ tends to infinity. Hence, there exist some number $\hat{n} \geq 2$ such that the incentive compatibility constraints starts binding.

The result stated above tells us that, while the consequences of imperfect electoral competition become milder when the number of candidates increases, the ex-post constraints needed to prevent candidates' moral hazard become more and more demanding. With more numerous competing candidates, in fact, the probability of being elected falls even when the announced platform has been implemented during the office. The incentives to cheat, therefore, become stronger and stronger. As a consequence, an increase in the number of candidates cannot determine a fall in political rents as in the full commitment case.

\section{Concluding remarks}

We have analysed how electoral competition constraints the ability of candidates to gain political rents. We have shown that complete rent dissipation can occur even when candidates don't observe perfectly voters' preferences and behaviour, as long as they know the mean of voters' heterogeneity distribution. In this case the candidates are able to reconstruct which is the median platform, and competition is Bertrand-type. If however there is aggregate uncertainty, i.e. the mean of the distribution of voters' heterogeneity is not observed and the median platform is not known, competition is relaxed even under a majority voting and winner-takes-all rule. In this case, which seems quite realistic, electoral competition is not able to induce rent dissipation.

Since Downs' seminal contribution, models of electoral competition are rooted in the Hotelling tradition of spatial competition/product differentiation. However, we have found a major difference between these two parallel problems. In a probabilistic model of product differentiation, the existence of consumers' heterogenity per se, even when the mean of their distribution is known, is sufficient to obtain positive profits in equilibrium, which increase in consumers' dispersion around the mean. This probabilistic model can be replicated in the more traditional address approach, in which consumers' heterogeneity corresponds to a distribution of "addresses" or ideal versions of the product in the space of varieties; in this framework, it is well known that the firms tend to offer differentiated products to relax price competition and obtain higher profits.

In our probabilistic voting model, voters' heterogenity per se does not 
play any role in equilibrium under a majority voting and winner-takes-all rule; it is the uncertainty on the mean of this distribution, what we defined aggregate uncertainty, that can relax electoral competition and allows positive rents. Hence, even if the candidates can offer diverging platforms, for instance by adding an ideological dimension to their policy, they find it convenient to converge to the median voter in the attempt to gain a lead in the campain.

Finally, we have shown that under certain conditions on the distribution of voters' heterogeneity, increasing the number of candidates may reduce political rents. However, abandoning the full commitment assumption and considering the ex-post incentives to prevent candidates' moral hazard once in office, an increase in the number of candidates tends to increase political rents.

\section{References}

[1] Anderson S., De Palma A., Thisse J.F., (1991), Discrete Choice Theory of Product Differentiation, MIT Press.

[2] Arrow K. (1990), Foreward to Enelow J. Hinich M. Advances in the Spatial Theory of Voting, Cambridge U.P.

[3] Enelow J., Hinich M. (1982), Nonspatial Candidate Characteristics and Electoral Competition, Journal of Politics, 44, 115-130.

[4] Ferejohn J. (1986), Incumbent Performance and Electoral Control, Public Choice, 50, 5-26.

[5] Grillo M., Polo M. (1993), Political Exchange and the Allocation of Surpuls: a Model of Two-Party Competition,in Breton A., Galeotti G. Salmon P. Wintrobe R. (eds.), Preferences and Democracy Kluwer.

[6] Laffont J.J. (1989), Fundamentals of Public Economics, MIT Press.

[7] Lindbeck A., Weibull J. (1987), A Model of Political Equilibrium in a Representative Democracy, Journal of Public Economics, 51, 195-209.

[8] Myerson R. (1993a), Review of J.Enelow and M.Hinich (1990) Advances in the Spatial Theory of Voting, Journal of Economic Literature, XXXI, $237-40$. 
[9] Myerson R. (1993b) Effectiveness of Electoral Systems for Reducing Government Corruption: a Game Theoretic Analysis, Games and Economic Behaviour, 5, 118-32.

[10] Persson T., Roland G., Tabellini G. (1997), Separation of Power and Political Accountability, Quarterly Journal of Economics, forthcoming.

[11] Persson T., Tabellini G. (1998a), Political Economics and Public Finance, in: A.Auerbach M.Feldstein (eds.), Handbook of Public Economics forthcoming.

[12] Persson T., Tabellini G. (1998b), The Size and Scope of Government: Comparative Politics and Rational Politicians, Marshall Lecture, 1998 EEA Congress, Berlin.

[13] Stigler G. (1972), Economic Performance and Political Competition, Public Choice, 91-106.

[14] Svennson J. (1997), The Control of Public Policy: Electoral Competition, Polarization and Primary Elections, mimeo

[15] Wittman D. (1973), Parties as Utility Maximizers, American Political Science Review, 490-98.

[16] Wittman D. (1977), Candidates with Policy Preferences: a Dynamic Model, Journal of Economic Theory, 180-89.

[17] ittman D. (1989), Why Democracies Produce Efficient Results, Journal of Political Economy, 97, 1395-424.

[18] Wittman D. (1990) Spatial Strategies when Candidates Have Policy Preferences, in Enelow J., Hinich M. (eds.) Advances in the Spatial Theory of Voting, Cambridge U.P. 